\title{
Una causa inusuale di ematuria
}

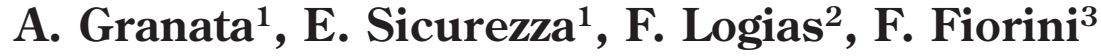 \\ ${ }^{1}$ U.O. di Nefrologia e Dialisi, A.O. "Vittorio Emanuele ", Catania \\ ${ }^{2}$ U.O. di Nefrologia e Dialisi, "Distretto Sanitario" Sorgono-Nuoro \\ ${ }^{3}$ U.O. di Nefrologia e Dialisi, Sanremo, ASL1 Imperiese, Imperia
}

\section{Caso clinico}

Una donna di 18 anni era ricoverata presso la nostra U.O. per accertamenti in merito al riscontro di microematuria a più controlli dell'esame urine. La storia clinica era silente e veniva solo segnalata la presenza di dispareunia. L'esame obiettivo non segnalava segni patologici. Gli esami ematici non rivelavano alterazioni: VES 4, GB $5600 \mathrm{ml}, \mathrm{Hb}$ 13.4\%, PTL $160000 \mathrm{ml}$, creatininemia $0.7 \mathrm{mg} / \mathrm{dl}$, glicemia 84 mg/dL; IgG, IgA, IgM, C3, C4, ANA, ENA, ANCA, FR nella norma. L'esame delle urine evidenziava microematuria e proteinuria non nefrosica (0.700 - $0.950 \mathrm{gr} / \mathrm{die})$, mentre l'urinocoltura era negativa. L'esame ecografico renovescicale dimostrava reni di diametro interpolare massimo di 107 e $118 \mathrm{~mm}$ a destra e sinistra rispettivamente e vescica nella norma, mentre l'ecografia endovaginale evidenziava varicocele pelvico sinistro. Veniva inoltre eseguito ecocolorDoppler dei vasi renali che non rilevava anomalie per ciò che riguarda i vasi arteriosi sia intraparenchimali che principali, mentre a livello della vena renale sinistra (VRS) evidenziava un lume particolarmente ristretto in corrispondenza del compasso aorta/arteria mesenterica superiore (AMS). Il diametro anteroposteriore della VRS era di $12.8 \mathrm{~mm}$ a livello ilare e di $2.1 \mathrm{~mm}$ a livello della porzione ristretta e il rapporto fra i due diametri era 6.10 (Fig. 1). Le velocità di picco sistolico nella VRS in sede ilare e aortomesenterica erano rispettivamente di $19 \mathrm{~cm} / \mathrm{s}$ e $87 \mathrm{~cm} / \mathrm{s}$. L'angio-TC confermava la stenosi della VRS (Fig. 2). Veniva pertanto posta diagnosi di "Nutcracker Syndrome".

Veniva effettuato il cateterismo della VRS secondo Seldinger attraverso l'approccio femorale destro al fine di misurare il gradiente pressorio fra la VRS e la vena cava inferiore a livello dell'aorta e dell'AMS: il valore riscontrato era di $6 \mathrm{mmHg}$ (valori normali $0 \mathrm{mmHg}$ ). La venografia confermava il varicocele ovarico sinistro con marcato flusso retrogrado (Fig. 3).
Uno stent al nitonolo auto-espandibile di $14 \times 40 \mathrm{~mm}(\mathrm{Lu}-$ minex, C.R. Bard Angiomed GmbH \& Co. Karlsruhe, De) era posizionato a livello della stenosi della VRS tramite filo guida previo posizionamento di dilatatore (Amplatz super stiff, 0.035”, Boston Scientific International, France) (Fig. 4).

La venografia post-procedurale dimostrava normale flusso attraverso la VRS in assenza di gradiente pressorio fra VRS e vena cava inferiore, mentre la cavografia rivelava corretto posizionamento dello stent con assenza di protrusione dello stesso in vena cava (Fig. 3). Al fine di prevenire la trombosi venosa si è somministrata eparina per due giorni, seguita da terapia cronica con acido acetilsalicilico. A distanza di dieci mesi dal posizionamento del-

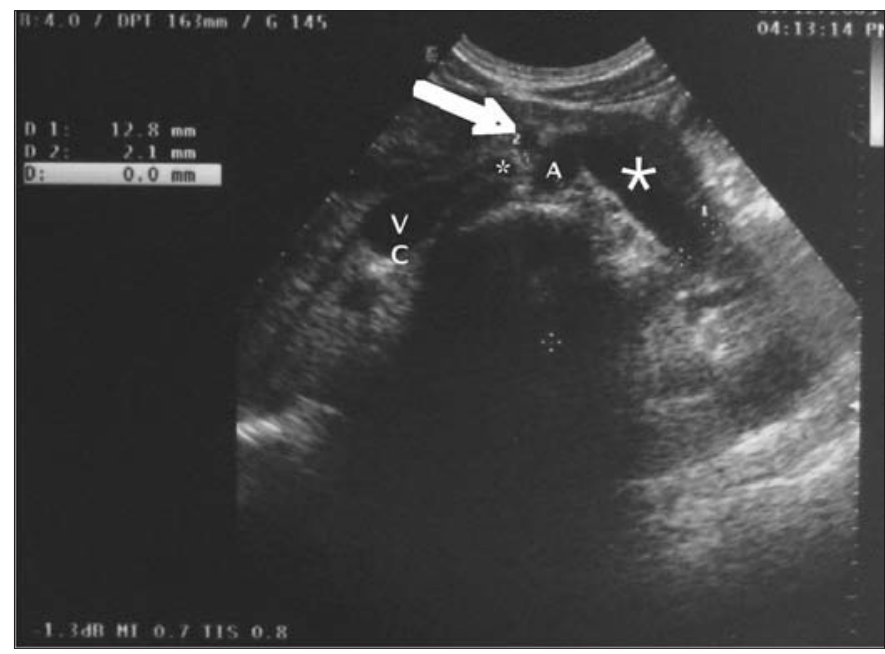

Fig. 1 - L'ecocolorDoppler rivela come la vena renale sinistra (asterisco) sia intrappolata fra aorta (A) e arteria mesenterica superiore (freccia): da notare la dilatazione del tratto sinistro della vena renale sinistra (asterisco grande) rispetto al tratto destro (asterisco piccolo). $\mathrm{VC}=$ vena cava inferiore. 


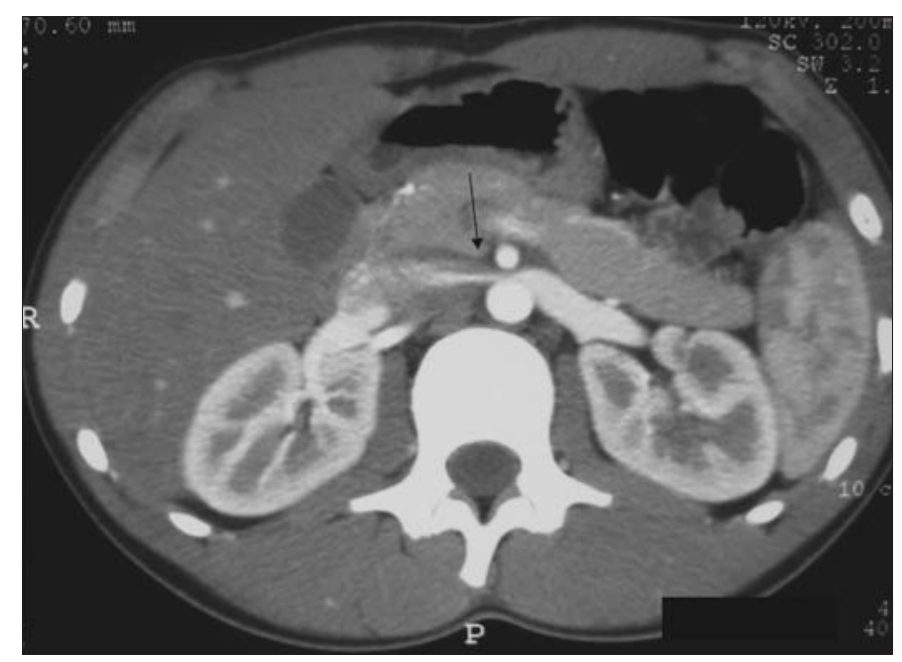

Fig. 2 - La TC con MdC evidenzia stenosi della vena renale sinistra con jet di contrasto attraverso il lume (freccia).

lo stent, la Paziente è asintomatica e libera da microematuria: il colorDoppler ha dimostrato normalità dei flussi renocavali.

\section{Discussione}

Il "nutcracker phenomenon" è solitamente definito come l'intrappolamento della VRS nella forchetta compresa fra l'aorta e l'AMS: è stato descritto per la prima volta da Schepper nel 1972. In modo simile è stata definita come "posterior nutcracker syndrome" l'ipertensione venosa secondaria a compressione della VRS intrappolata fra l'aorta addominale e la colonna vertebrale.

La diagnosi differenziale deve essere posta fra la dilatazione asintomatica della VRS, "nutcracker phenomenon", e la "nutcracker sindrome (NS)", quest'ultima in presenza di micro/macroematuria, varicocele e dolore lombare o addominale non spiegabile. La NS è causata da compressione della VRS fra l'AMS e l'aorta, con conseguente ipertensione retrograda sulla VRS $(1,2)$. Lipertensione venosa renale riscontrata nei pazienti con NS porta a sviluppo di circoli venosi collaterali sul circolo pelvico che può causare ematuria e flusso retrogrado nella vena gonadica. La sindrome si presenta sia nei maschi che nelle femmine e viene classificata sulla base della posizione delle valvole venose competenti bypassate come varicocele lombare e pelvico. Nei maschi il varicocele può causare disordini della spermatogenesi, dolore scrotale, mentre nelle femmine può essere presente sindrome da congestione pelvica, ben caratterizzata da dolore post-coitale ed algia del punto ovarico: la

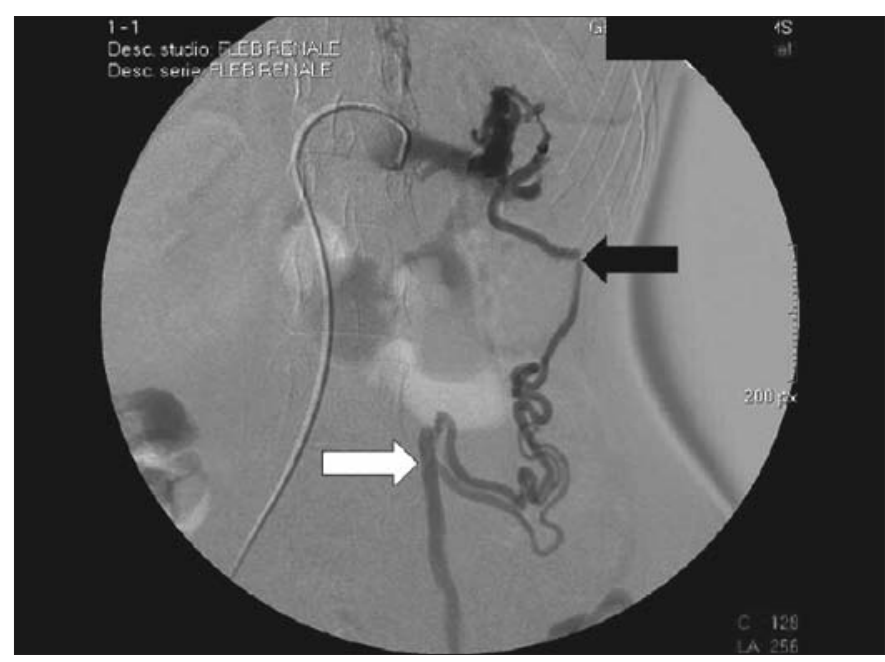

Fig. 3 - La venografia dimostra varicocele ovarico (freccia bianca) con aumentato flusso retrogrado (freccia nera).

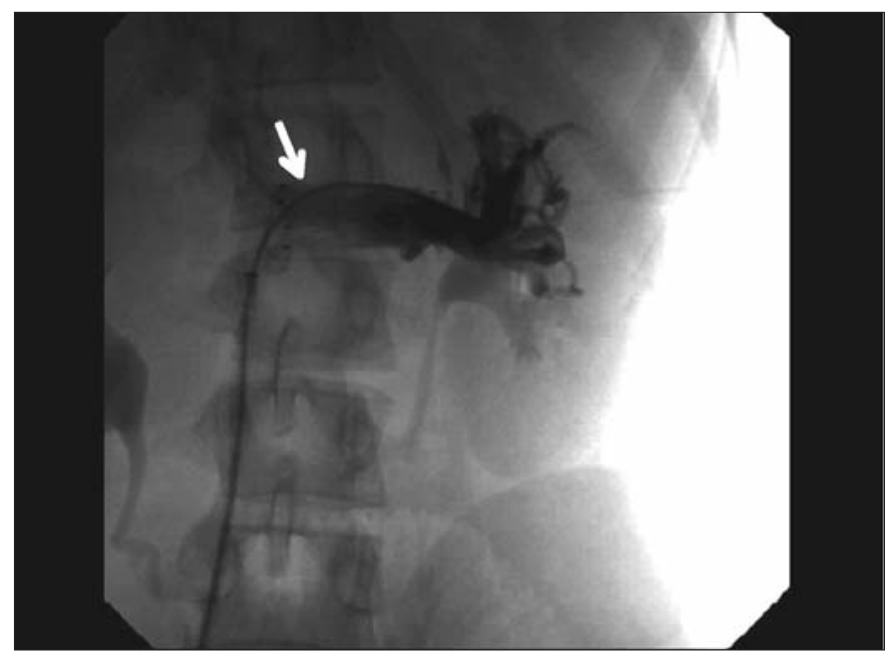

Fig. 4 - Venografia post-procedurale: la freccia indica lo stent posizionato in vena renale sinistra.

paziente può comunque manifestare sintomatologia varia e aspecifica (3). Altri segni riportati in letteratura sono dolore al fianco, sindrome da congestione pelvica e varici atipiche all'arto inferiore con sintomi da insufficienza venosa.

La diagnostica per immagini può giovarsi sia dell'ecocolorDoppler che della Tomografia Computerizzata che della Risonanza Magnetica, ma la diagnosi definitiva è posta solo attraverso la flebografia con manometria della VRS e della vena cava inferiore (4).

L'embolizzazione transcateterale della vena ovarica può essere necessaria per il trattamento della sindrome da con- 
gestione pelvica con un successo clinico iniziale compreso fra l' $88 \%$ e il $100 \%(5,6)$, mentre il beneficio a lungo termine varia fra il $68 \%$ e il $75 \%$ delle pazienti trattate $(7,8)$. Tuttavia nelle pazienti con NS la sintomatologia può ricomparire a causa della persistenza dell'ipertensione venosa renale. Numerosa è la tipologia di procedure chirurgiche (da mininvasive a endovascolari) proposte per ridurre la compressione della VRS (1). In letteratura è ancora basso il numero di trattamenti della NS attraverso il posizionamento di stent endovasali (9-13). Segawa et al (9) hanno riportato per primi nel 1999 il posizionamento di stent endovenoso in una donna di 40 anni con micro/macroematuria asintomatica.

Nel nostro caso la giovane età della paziente ha determinato la scelta di non intervenire sulla vena ovarica prossimale e di tentare direttamente lo stenting della VRS. A distanza di dieci mesi dalla procedure, la paziente appare libera da ematuria e dispareunia.

antonio.granata4@tin.it

f.fiorini@asl1.liguria.it

\section{Bibliografia}

1. Byoung-Soo Cho, Young-Mi Choi, Hyeon-Ho Kang, Seong Jin Park, Joo Won Lim and Tai Young Yoon. Diagnosis of nut-cracker phenomenon using renal Doppler ultrasound in orthostatic proteinuria. Nephrol Dial Transplant 2001; 16: 1620-5.

2. Rudloff U, Holmes RJ, Prem JT, Faust GR, Moldwin R, Siegel D. Mesoaortic compression of the left renal vein (nutcracker syndrome): case reports and review of the literature. Ann Vasc Surg 2006; 20 (1): 120-9.

3. Marsman JW. The aberrantly fed varicocele: frequency, venographic appearance, and results of transcatheter embolization. AJR Am J Roentgenol 1995; 164(3): 649-57.

4. Ahmed K, Sampath R, Khan MS. Current trends in the diagnosis and management of renal nutcracker syndrome: a review. Eur J Vasc Endovasc Surg 2006; 31(4): 410-6.

5. Cordts PR, Eclavea A, Buckley PJ, DeMaioribus CA, Cockerill ML, Yeager TD. Pelvic congestion syndrome: early clinical results after transcatheter ovarian vein embolization. J Vasc Surg 1998; 28(5): 862-8.

6. Venbrux AC, Chang AH, Kim HS, et al. Pelvic congestion syndrome (pelvic venous incompetence): impact of ovarian and internal iliac vein embolotherapy on menstrual cycle and chronic pelvic pain. J Vasc Interv Radiol 2002; 13: 171-8.
7. Maleux G, Stockx L, Wilms G, Marchal G. Ovarian vein embolization for the treatment of pelvic congestion syndrome: long-term technical and clinical results. J Vasc Interv Radiol 2000; 11(7): 859-64.

8. d'Archambeau O, Maes M, De Schepper AM. The pelvic congestion syndrome: role of the "nutcracker phenomenon" and results of endovascular treatment. JBR-BTR 2004; 87(1): 1-8.

9. Segawa N, Azuma H, Iwamoto Y, et al. Expandable metallic stent placement for nutcracker phenomenon. Urology 1999; 53: 631-3.

10. Scultetus AH, Villavicencio JL, Gillespie DL. The nutcracker syndrome: its role in the pelvic venous disorders. J Vasc Surg 2001; 34: 812-9.

11. Kim SJ, Kim CW, Kim S, et al. Long-term follow-up after endovascular stent placement for treatment of nutcracker syndrome. J Vasc Interv Radiol 2005; 16(3): 428-31.

12. van der Laan L, Vos JA, de Boer E, van den Berg JC, Moll FL. The central-venous compression syndrome: rare, but adequately treatable with endovascular stenting. Ned Tijdschr Geneeskd 2004; 148: 433-7.

13. Hartung O, Grisoli D, Boufi M, et al. Endovascular stenting in the treatment of pelvic vein congestion caused by nutcracker syndrome: lessons learned from the first five cases. J Vasc Surg 2005; 42(2): $275-80$. 